\title{
USO DE MICRONUTRIENTES Y GRADO DE ANEMIA EN NIÑOS MENORES DE 3 AÑOS ATENDIDOS EN UN CENTRO DE SALUD ICA 2017
}

\author{
USE OF MICRONUTRIENTS AND DEGREE OF ANEMIA IN CHILDREN UNDER 3 \\ YEARS OLD ATTENDED AT A CENTER HEALTH ICA 2017
}

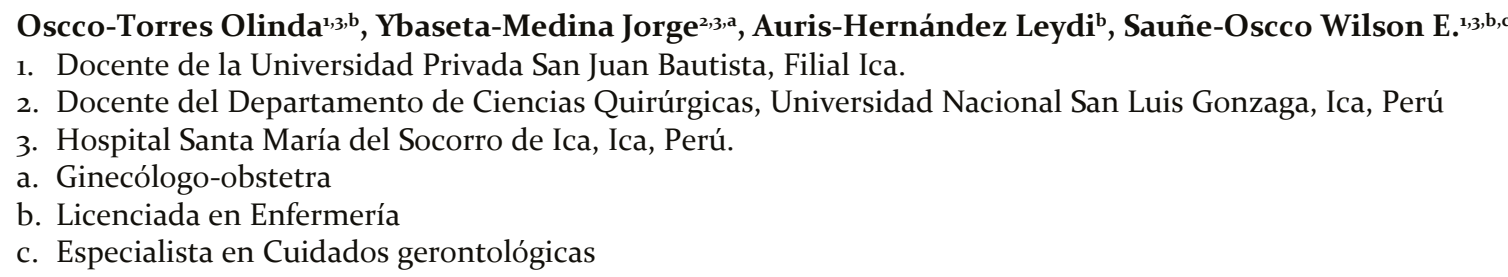

\section{Correspondencia:}

Ybaseta-Medina Jorge.

Dirección: Villa del Médico B-7 Ica,

Perú

Número de celular: +51956747941

Correo Electrónico:

jorgeybaseta@yahoo.es

http://orcid.org/0000-0003-1224-1 357

\section{Contribuciones De Autoría:}

OTO, YMJ, AHL, SOWE

participaron en el diseño del estudio, el análisis de los datos, revisaron críticamente el artículo y aprobaron la versión final.

Conflicto De Intereses: No declarados.

Financiamiento: Autofinanciado.

\section{Como Citar}

Olinda Oscco Torres, Jorge Ybaseta Medina, Leydi Auris Hernández, Wilson E. Sauñe Oscco. Uso de micronutrientes y grado de anemia en niños menores de 3 años atendidos en un centro de salud Ica 2017. Rev méd panacea. 2019; 8(2): 69-72. doi: https://doi.org/10.35563/rmp.v8 i2.273

Recibido: 23 - 03 - 2019

Aceptado: 18 - 05 - 2019

Publicado: 12 - 05 - 2019

\section{RESUMEN}

Objetivo: Determinar el uso de los micronutrientes y grado de anemia en niños menores de 3 años atendidos en un Centro de Salud de Ica 2017. Materiales y métodos: El estudio fue cuantitativo, de tipo descriptivo, y transversal, la muestra estuvo conformada por 40 niños menores de tres años con sus respectivas madres, la técnica que se utilizó fue la observación y el análisis documental. Resultados: En relación a los datos generales de la madre, el $47.5 \%$ (19) tienen 19 a 34 años, el 50\% (20) tienen instrucción superior, el $40 \%$ (16) son solteras y el $57.5 \%$ (23) trabaja fuera de casa; sobre los datos del niño, el 55\% (22) tienen de 6 a 12 meses, el 72.5\% (29) recibió solo lactancia materna hasta los seis meses y el $100 \%$ (40) inició la alimentación complementaria a los 6 meses. El uso de micronutrientes es adecuado en el $82.5 \%$ (33) de las madres y es inadecuado en el $17.5 \%(07)$. Según valores de hemoglobina, el $62.5 \%$ (25) de los niños no presentan anemia, el $37.5 \%$ (15) presentan anemia leve, no encontrando niños con anemia moderada ni anemia severa. Conclusiones: El uso de micronutrientes que administran las madres a sus niños es adecuado y los niños no presentan anemia en su mayoría, existiendo porcentajes menores de niños con anemia leve por lo que se sugiere que se continúe con el abastecimiento continuo de los micronutrientes realizando campañas educativas a fin de comprometer a las madres en la administración correcta de los micronutrientes en sus menores hijos aprovechando los momentos de contacto con las madres para la educación respectiva en las sala de espera, durante sus controles de inmunizaciones, controles CRED, y realizar un monitoreo periódico del control de hemoglobina.

\section{Palabras clave: Micronutrientes, anemia, (Fuente: DeCS BIREME)}

\begin{abstract}
Objective: To determine the use of micronutrients and the degree of anemia in children under 3 years of age served in an Ica Health Center 2017. Materials and methods: The study was quantitative, descriptive, and cross-sectional, the sample consisted of 40 children under three years with their respective mothers, the technique used was observation and documentary analysis. Results: In relation to the general data of the mother, $47.5 \%$ (19) are 19 to 34 years old, $50 \%$ (20) have higher education, $40 \%$ (16) are single and 57.5\% (23) work ; On the data of the child, $55 \%$ (22) have from 6 to 12 months, $72.5 \%$ (29) received only breastfeeding until six months and $100 \%(40)$ started complementary feeding at 6 months. The use of micronutrients is adequate in $82.5 \%$ (33) of mothers and is inadequate in $17.5 \%$ (07). According to hemoglobin values, $62.5 \%$ (25) of the children do not have anemia, $37.5 \%$ (15) have mild anemia, and there are no children with moderate anemia or severe anemia. Conclusions: The use of micronutrients administered by mothers to their children is mostly adequate and children do not have anemia in their majority. There are smaller percentages of children with mild anemia, so it is suggested that continuous supply of the micronutrients conducting educational campaigns in order to engage mothers in the correct administration of micronutrients in their younger children, taking advantage of the moments of contact with mothers for the respective education in the waiting room, during their immunization controls, CRED controls, and perform periodic monitoring of hemoglobin control.
\end{abstract}




\section{INTRODUCCIÓN}

Según la Organización Mundial de la Salud (OMS), en el año 2016 la prevalencia de anemia afectó a 273.2 millones de niños, de los cuales la mayor prevalencia fue de $47,4 \%$ en los niños en edad lactante y preescolar los índices más fueron en África $67,6 \%$ y Asia Sudoriental $65,5 \%$, en el Mediterráneo Oriental, fue del $46 \%$, y del $20 \%$ aproximadamente en las demás regiones Las Américas, Europa y Pacífico Occidental. (1)

En América Latina y El Caribe la tasa de prevalencia de anemia en el 2016 en niños menores de cinco años fue del $29.3 \%$, lo cual corresponde a aproximadamente 23 millones de niños afectados sin diferenciar estrato socio-económico, grupo étnico cultural o área de residencia. (2)

En relación al uso de los micronutrientes, en el 2013 en Guatemala se halló un bajo porcentaje a la suplementación con micronutrientes espolvoreados en las comunidades de Colmenas y Suchiquer con $40 \%$ y $43 \%$. (3)

En el 2013 en Ecuador, el 50\% del total de los niños de los Centros Infantiles del Buen Vivir (CIBV), dejó de consumir Chispas a la segunda semana de intervención a consecuencia que los padres acordaron con las promotoras suspender dicho tratamiento ya que los infantes en mención presentaron diarrea a partir de su consumo. (4)

Según la Encuesta Demográfica y de Salud Familiar (ENDES), en el Perú en el primer semestre del 2016 los niños menores de 3 años cursaron una prevalencia de anemia de $52.3 \%$ en la zona rural y $40.2 \%$ en la zona urbana. Lima se vio afectada en un $35.5 \%$ y en el primer semestre del 2016 aproximadamente 149 mil niños y niñas de 6 a menos de 36 meses presentaron anemia. (5) El consumo de hierro en el grupo de edad de 6 a menos de 36 meses es deficiente y la causa de este consumo inadecuado es multifactorial, la dieta no provee la cantidad necesaria para cubrir los requerimientos de este mineral, alcanzando a cubrir solo un $62.9 \%$ en el caso de los niños menores de dos años. (6)

En tal sentido el objetivo de la investigación fue determinar el uso de los micronutrientes y el grado de anemia en niños menores de 3 años atendidos en un en un Centro de Salud de Ica 2017.

\section{MATERIALES Y MÉTODOS}

Se realizó una investigación cuantitativa, descriptiva y de corte transversal, haciendo uso del muestreo no probabilístico intencional, considerándose a la población de 81 niños menores de 3 años, y la muestra fue de 40 niños y sus madres. Para la recolección de datos de la primera variable se realizó la observación la cual se hizo durante la visita domiciliaria a la madre que previamente asistió al control de CRED de su niño; para la segunda variable se hizo uso de la historia clínica. Previo a la aplicación del instrumento de recolección de datos se sometió a control de calidad para determinar su validez, a través de la concordancia de opinión de Jueces expertos, obteniendo una validez de 0.022 ; posteriormente se demostró la confiabilidad del instrumento de la primera variable a través del Alfa de Cronbach con un resultado de 0.6 .

El análisis estadístico de los datos se realizó haciendo uso del paquete informático Microsoft Excel y Microsoft Office Word 2007.

\section{RESULTADOS}

Según datos generales de las madres el 47.5\% (19/40) tienen entre 19 a 34 años, el 50\% (20/40) tienen instrucción superior, el $40 \%$ (16/40) son solteras y el $57.5 \%(23 / 40)$ trabaja fuera de casa; según datos del niño, el 55\% (22/20) tienen de 6 a 12 meses, el 72.5\% (29/40) recibió solo lactancia materna hasta los seis meses y el 100\% (40/40) inició la alimentación complementaria a los 6 meses.

Respecto al uso de micronutrientes cuando la madre les administra a sus niños es adecuado en el $82.5 \%$ (33/40) e inadecuado en el 17.5\% (07/40)

El grado de anemia según valores de hemoglobina se tiene que el $62.5 \%(25 / 40)$ de los niños no presentan anemia, 37.5\% (15/40) presentan anemia leve, no encontrando niños con anemia moderada ni anemia severa.

Tabla 1: Datos generales de las madres y niños menores de 3 años atendidos en el Centro de Salud de Tate, Ica 2017.

\begin{tabular}{|c|c|c|c|}
\hline DATOS GENERALES & CATEGORÍA & Fr. & $\%$ \\
\hline \multirow[t]{3}{*}{ Edad de la madre } & Menor de 19 años & 6 & 15.0 \\
\hline & 19 a 34 años & 19 & 47.5 \\
\hline & Más de 34 años & 15 & 37.5 \\
\hline \multirow[t]{3}{*}{ Grado de instrucción } & Primaria & 2 & 05.0 \\
\hline & Secundaria & 18 & 45.0 \\
\hline & Superior & 20 & 50.0 \\
\hline \multirow[t]{4}{*}{ Estado civil } & Soltera & 16 & 40.0 \\
\hline & Casada & 14 & 35.0 \\
\hline & Conviviente & 8 & 20.0 \\
\hline & Divorciada & 2 & 05.0 \\
\hline \multirow[t]{2}{*}{ Ocupación } & Trabaja & 23 & 57.5 \\
\hline & No trabaja & 17 & 42.5 \\
\hline \multicolumn{4}{|l|}{ DATOS DEL NIÑO } \\
\hline \multirow[t]{3}{*}{ Edad } & 6 a 12 meses & 22 & 55.0 \\
\hline & 13 a 24 meses & 17 & 42.5 \\
\hline & 25 a 36 meses & 1 & 02.5 \\
\hline \multirow[t]{2}{*}{ Solo lactancia materna } & $\mathrm{Si}$ & 29 & 72.5 \\
\hline & No & 11 & 27.5 \\
\hline \multirow{2}{*}{$\begin{array}{l}\text { Inicio de alimentación } \\
\text { complementaria }\end{array}$} & 6 meses & 40 & 100.0 \\
\hline & Otras edades & 0 & 0 \\
\hline Total general & & 40 & 100.0 \\
\hline
\end{tabular}




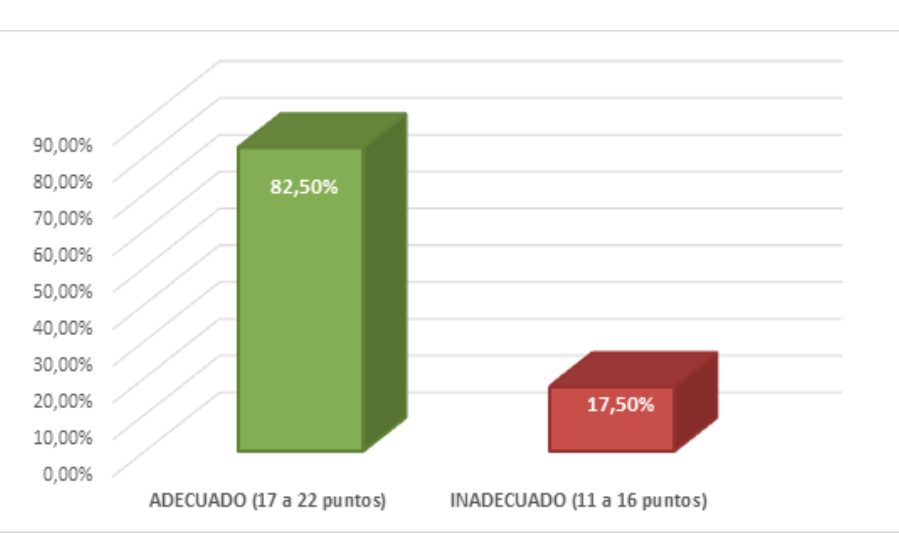

Gráfico 1: Consolidado global respecto al Uso de los micronutrientes en niños menores de 3 años atendidos en el Centro de Salud de Tate, Ica 2017.

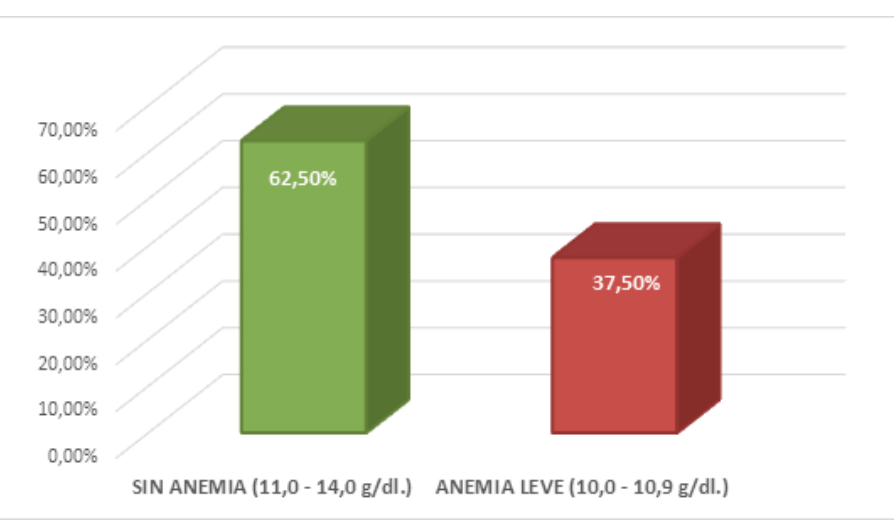

Gráfico 2: Grado de anemia según valores de hemoglobina en niños menores de 3 años atendidos en el Centro de Salud de Tate, Ica 2017.

\section{DISCUSIÓN}

Referente a los datos generales de las madres y sus niños menores de 3 años, son contrarios con Farfán A. (7) quién encontró un bajo nível de escolaridad en las madres; Junco J. (8) halló que el $42,9 \%$ son madres jóvenes entre 17 y 25 años de edad, el $94.4 \%$ de las madres jóvenes solo alcanza el grado instrucción primaria, del total de las madres. Los datos demográficos de las madres como la edad, grado de instrucción, ocupación, son factores que intervienen en la administración de los micronutrientes, el grado de instrucción superior en las madres hallado en la investigación juega un papel importante debido a un mayor conocimiento sobre la alimentación del niño en cambio si la madre trabaja fuera de casa es perjudicial porque no brinda un cuidado directo al niño, por lo que se deben considerar estos aspectos al momento de la orientación y recomendación en el uso de los micronutrientes.

Respecto al consolidado global sobre el uso de los micronutrientes, los resultados son contrarios con los obtenidos por Santisteban L, Valdiviezo M. (9) respecto a la adherencia con micronutrientes, donde solo un $58.9 \%$ de niños menores de 36 meses presentan adherencia al tratamiento con micronutrientes; Izquierdo C. (10) halló que el consumo de micronutrientes es regular em $46.97 \%, 30.30 \%$ el nivel es malo y el $22.73 \%$ su nivel de consumo es bueno. Estos resultados permitem inferir que el uso de los micronutrientes utilizados por las madres para el consumo de sus niños son inadecuados en porcentajes considerables por lo que es necesario mejorar las estrategias para orientar y concientizar a las madres respecto al uso adecuado de la suplementación con micronutrientes.

Referente al grado de anemia según valores de hemoglobina en los niños menores de 3 años, resultados similares con Izquierdo C. (10) donde los resultados de los niños entre las edades de 6 a 36 meses que reciben micronutrientes el $96.97 \%$ se encuentran con valores normales de hemoglobina y un 3.03\% con anemia leve; así mismo nuestros resultados son contrarios con Santisteban L, Valdiviezo M. (9) quien halló que solo el $37,5 \%$ tienen un nivel de hemoglobina normal de $11 \mathrm{~g} / \mathrm{dl}$. La Anemia continúa siendo un problema de salud pública en el Perú, se observa en todos los estratos sociales y con mayor frecuencia en los niños menores de cinco años, por lo que es importante la implementación de estrategias enseñándole a la madre que debe incorporar alimentos que contengan hierro como la sangrecita, bazo, riñones, hígado, carnes rojas y pescados oscuros, dando énfasis a la suplementación con micronutrientes cumpliendo la conservación y administración adecuada para obtener los beneficios deseados. 


\section{REFERENCIAS BIBLIOGRÁFICAS}

1. OMS. Anemia ferropénica: Investigación para soluciones eficientes y viables; 2016 Disponible en: : / / w w w 2. pa ho.org/h q/ind ex.ph p? option =com_content $\&$ view $=$ article $\& i d=11679 \% 3$ Airo n-deficiency-anemia-research-on-iron- es.

2. FAO. Organización Regional para América Latina y el Caribe. Panorama de la seguridad alimentaria y nutricional, 2016.

3. Farfán A. Adherencia de las madres a la suplementación de niños de 6 a 59 meses de edad, con micronutrientes espolvoreados, en las comunidades Suchiquer y Colmenas del municipio de Jocotán, Chiquimula. Chiquimula: Universidad de San Carlos de Guatemala. Facultad de Ciencias Químicas y Farmacia.

4. Reyes M. Evaluación del consumo y tolerancia del suplemento Chispaz en los CIBV de los barrios San Pedro y La Loma de la comunidad de Cangahua, octubre a noviembre 2012. Quito: Pontificia Universidad Católica del Ecuador.

5. Instituto Nacional de Estadística e Informática (INEI). Perú: Indicadores de resultados de los Programas Estratégicos, 2016. Encuesta Demográfica y de Salud Familiar - ENDES Continúa. Lima: INEl; 2016.
6. INEI. 948 mil niños menores de cinco años tienen anemia en Perú. URL. Disponible en: http://rpp.pe/economia/economia/inei.

7. Farfán A. Adherencia de las madres a la suplementación de niños de 6 a 59 meses de edad, con micronutrientes espolvoreados, en las comunidades Suchiquer y Colmenas del Municipio de Jocotán, Chiquimula, Guatemala. 2013.

8. Junco J. Identificación de los factores que contribuyen y limitan la efectividad del programa de suplementación con multimicronutrientes en la reducción de la anemia de niños menores de tres años del ámbito rural de Vinchos de Ayacucho. 2016.

9. Santisteban L, Valdiviezo M. Relación entre la adherencia al tratamiento con micronutrientes y el nivel de hemoglobina en los niños menores de 36 meses del Centro de Salud San Martin Lambayeque - Perú. 2017.

10. Izquierdo C. Influencia del consumo de micronutrientes en la prevención y tratamiento de anemia leve en niños de 6 a 36 meses. Centro de Salud Alto Perú -Sausal- La Libertad Trujillo - Perú. 2016 Disponible en: repositorio.ucv.edu.pe/handle/UCV/772? show=full.

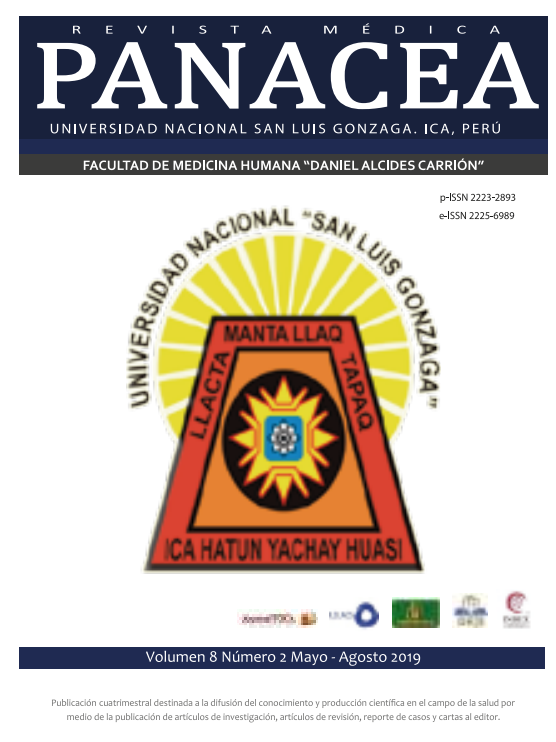

